УДК 519.21

\title{
Chaotic Dynamics of a Three-dimensional Endomorphism
}

\author{
Hacene Gharout* \\ Nourredine Akroune ${ }^{\dagger}$ \\ Laboratoire des Mathématiques Appliquées \\ Faculté des Sciences Exactes \\ Université de Bejaia, Bejaia, 06000 \\ Algerie \\ Abelkadous Taha ${ }^{\ddagger}$ \\ INSA, University of Toulouse \\ 135 Avenue de Rangueil, Toulouse, 31077 \\ France \\ Daniele-Fournier Prunaret ${ }^{\S}$ \\ Laboratory of LAAS-CNRS \\ INSA, University of Toulouse \\ 7 Avenue du Colonel Roche, Toulouse, 31077
}

France

Received 26.02.2018, received in revised form 09.07.2018, accepted 10.09.2018

The present work describes the phases plane bifurcations of some attractors given by a noninvertible three-dimensional map. This study is conducted through the critical manifolds concepts, generalization of critical points and critical lines introduced by Gumowski and Mira [1,2]. The phase plane shared within two open regions: the first (denoted $Z_{0}$ ) each point having no real preimage, and the second (denoted $Z_{2}$ ) each point having two real preimages. The regions $Z_{0}, Z_{2}$ are separated by the critical manifolds, locus of points having two coincident preimages. This requires the visualization of critical manifolds in the three dimensional phases space. And this work also describes the passage of invariant or attractor curves towards weakly chaotic attractors then towards hyper-chaotic attractors via the contact bifurcation through the critical manifolds, which disappear after the contact bifurcation with the its attraction basin boundary.

Keywords: critical manifold, closed invariant curve, weakly chaos, chaos.

DOI: $10.17516 / 1997-1397-2019-12-1-36-50$.

\section{Introduction and preliminaries}

The aim of this work is to study the dynamic of a real three dimensional noninvertible nonlinear map and its phases space. Critical manifolds will be illustrated in the case of three dimensions, concepts which generalize the critical points and critical lines defined studied in dimension one and two [3-9]. The three dimensional noninvertible map is given by:

$$
T\left\{\begin{array}{l}
x_{n+1}=y_{n}, \\
y_{n+1}=z_{n}, \\
z_{n+1}=x_{n}^{2}+a y_{n}\left(x_{n}+z_{n}\right)+b .
\end{array}\right.
$$

where $a$ and $b$ are two real parameters.

\footnotetext{
*gharouthacene@gmail.com

†akroune_n@yahoo.fr

†taha@insa-toulouse.fr

$\S$ daniele.fournier@insa-toulouse.fr

(c) Siberian Federal University. All rights reserved
} 
The recurrence $T$ defined in (1), admits two fixed points $X_{1}=\left(x_{1}, x_{1}, x_{1}\right)$ and $X_{2}=$ $=\left(x_{2}, x_{2}, x_{2}\right)$, with:

$$
x_{1}=\frac{1+\sqrt{1-4 b-8 b a}}{2(1+2 a)}, \quad x_{2}=\frac{1-\sqrt{1-4 b-8 b a}}{2(1+2 a)}
$$

defined for:

$$
\begin{gathered}
a \neq \frac{-1}{2} \text { and } b \leqslant \frac{1}{4(2 a+1)} \text { if } a>\frac{-1}{2} ; \\
b \geqslant \frac{1}{4(2 a+1)} \text { if } a<\frac{-1}{2} .
\end{gathered}
$$

For $a=\frac{-1}{2}$, the map $T$ admits the unique fixed point, $X^{*}=(b, b, b)$.

The classical bifurcations obtained are:

Fold Bifurcation (Node-saddle): The equation of the Fold bifurcation curve, noted $\Lambda_{(1)^{\circ}}^{1}$, is $b=\frac{1}{4+8 a}$, with $x=\frac{1}{2+4 a}$ for $a \neq \frac{-1}{2}$.

Flip bifurcation (doubling period): The equation of the bifurcation Flip curve, noted $\Lambda_{1}^{1}$, is $b=\frac{-3}{4}-\frac{1}{2} a$, with $x=\frac{-1}{2}$.

Neimark-Sacker Bifurcation: The Neimark-Sacker bifurcation occurs when the modulus of the conjugated complex multipliers $S_{i}, i=1,2$ for a stable fixed point or a cycle of order k of $T$, crosses value 1 . The fixed point or the cycle becomes unstable, giving rise to a closed invariant curve which has the same stability as the fixed point or the original cycle. The obtained curves equations of Neimark-Sacker bifurcation are the following:

$$
\begin{aligned}
& \Gamma_{1}^{1}: \quad b=\frac{-2-3 a-2 a^{2}-2 a^{3}+2 \sqrt{4+2 a-7 a^{2}-4 a^{3}+2 a^{4}+2 a^{5}+a^{6}}}{8+8 a+2 a^{2}}, \\
& \Gamma_{2}^{1}: \quad b=\frac{-2-3 a-2 a^{2}-2 a^{3}-2 \sqrt{4+2 a-7 a^{2}-4 a^{3}+2 a^{4}+2 a^{5}+a^{6}}}{8+8 a+2 a^{2}} .
\end{aligned}
$$

In order to analyse the local stability of the fixed point, we need to evaluate the jacobian matrix at the fixed point and calculate the eigenvalues. Unfortunately the expressions defining the eigenvalues are so complicated that nothing can be said analytically. We can still say something through numerical simulations. After several numerical computations, we have found a generic two-dimensional bifurcation diagram in the $(\mathrm{a}, \mathrm{b})$ parameters plane, where the bifurcations curves (Fold $\left(\Lambda_{(1)^{o}}^{1}, \Lambda_{(1)^{\circ}}^{2}\right)$, Flip $\left(\Lambda_{1}^{1}\right)$ and Neimark-Sacker $\left(\Gamma_{1}^{1}, \Gamma_{2}^{1}\right)$ bifurcations curves) delimit the zone of stability of the fixed point $X_{2}$ of the map $T$ in the parameters plane $(a, b)$ (see Fig. 1, where the blue color is the $X_{2}$ stability area).

\section{Phases space of the map $T$}

\subsection{Critical manifolds of the map $T$}

In the case of the considered transformation $\mathrm{T}$, we note that the critical manifolds are surfaces in the three-dimensional space, and are parallel planes to one of the planes in space $(x, y, z)$, when the parameter $a$ vanishes. The critical curves, denoted $E C$, are surfaces divide the phases space in areas denoted $Z_{i}$, i integer, where each $Z_{i}$ corresponds to the set of points of the phase 
space that have a preimage of rank i for T. A critical range of rank $k+1$, denoted $E C_{k}$ is the manifold of rank k using $E C_{0}$ (see equation (5)), $k=1,2, \ldots\left(E C_{k}=T^{k}\left(E C_{0}\right)\right.$ for all $k \geqslant 1$ ). Representation of some critical manifolds for different values of $a$ and $b$ are presented in Fig. 2.

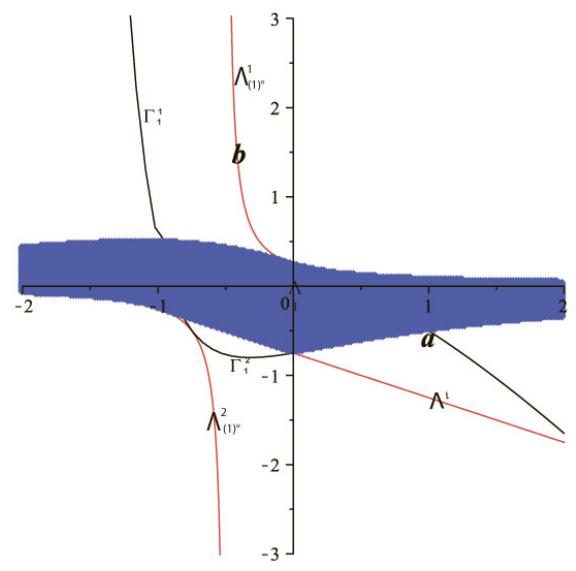

Fig. 1. The blue region is the stability zone of the fixed points of $\mathrm{T}$

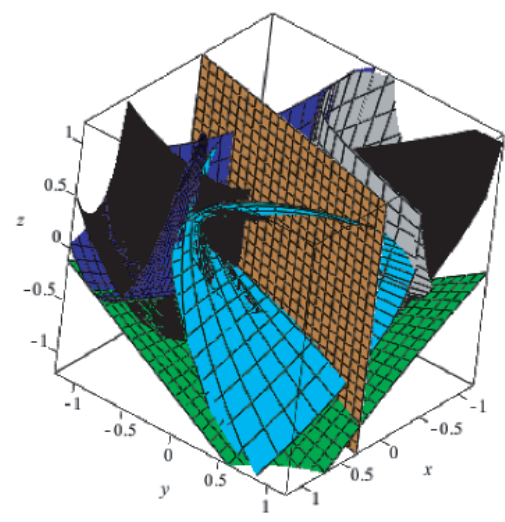

(a)

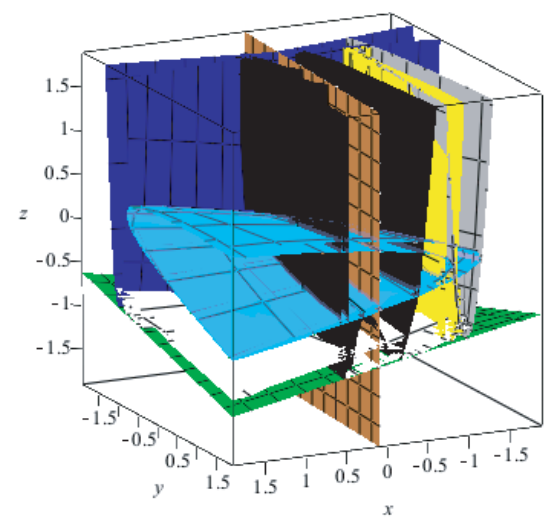

(b)

Fig. 2. Representation of some critical manifolds for different values of $a$ and $b$ at $(a, b)=$ $=(-0.56,-0.75)$ in the figure $(\mathrm{a})$ and at $(a, b)=(-0.1,-0.9758)$ in the figure (b). Color codes are: $E C_{-1}$ in brown color, $E C$ in green, $E C_{1}$ in blue, $E C_{2}$ in gray, $E C_{3}$ in cyan, $E C_{5}$ in black, $E C_{6}$ in white and $E C_{8}$ in yellow

The phases space are divided into several regions by the critical manifolds, each of them has a different number of preimages of rank one. $E C_{-1}$ is the antecedent of rank one of $E C_{0}$. The equation for the critical manifold $E C_{-1}$ for the map T satisfies $|J(x, y, z)|=0$, where $J(x, y, z)$ is the Jacobian of $\mathrm{T}$ at point $(x, y, z)$ :

$$
J(x, y, z)=\left(\begin{array}{ccc}
0 & 1 & 0 \\
0 & 0 & 1 \\
2 x+a y & a(x+z) & a y
\end{array}\right) .
$$

The obtained critical manifold $E C_{-1}$ depends only on $y, z$ and the parameter $a$, with

$$
x=-\frac{a}{2} y, \quad y \text { and } z \in \mathbb{R} .
$$


Therefore, $E C_{-1}$ is given by:

$$
E C_{-1}=\left\{\left(-\frac{a}{2} y, y, z\right), y, z \in \mathbb{R}\right\} .
$$

The manifold $E C_{0}$ noted $E C$ is defined by:

$$
E C_{0}=T\left(E C_{-1}\right)=\left\{\left(y, z, \frac{1}{4} a^{2} y^{2}+a y\left(-\frac{1}{2} a y+z\right)+b\right), y, z \in \mathbb{R}\right\} .
$$

The critical manifolds or the critical surfaces (cf. [10]), in our cas, are planes for $a$ equal to zero. A graphic illustration of the first eight critical planes is given in Fig. 3, where the critical manifolds are noted $P C_{n}$ instead of $E C_{n}$, with

$$
\begin{gathered}
P C_{-1}=\{(0, y, z), y, z \in \mathbb{R}\}, \\
P C_{0}=T\left(P C_{-1}\right)=\{(y, z, b), y, z \in \mathbb{R}\},
\end{gathered}
$$

and $P C_{n}=T^{n+1}\left(P C_{-1}\right)$, for $n \geqslant 0$.
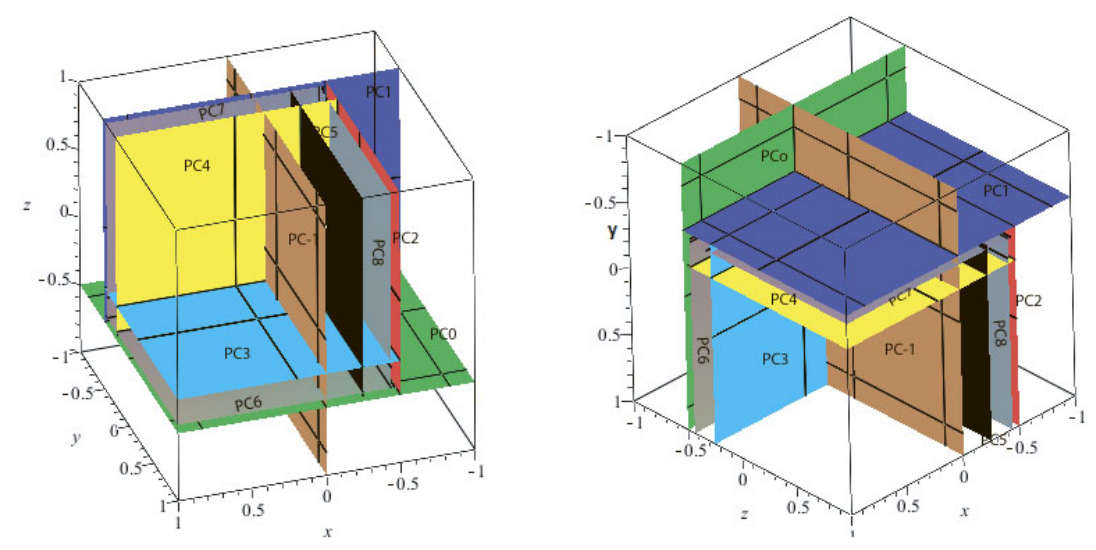

Fig. 3. The first eight critical planes of $T$ at $a=0$ and $b=-0.5$

$E C$ is a manifold separating the space $\mathbb{R}^{3}$ into two regions denoted $Z_{0}$ and $Z_{2}$. The region $Z_{2}$ contains all the points that have two distinct antecedents of rank 1 , and the region $Z_{0}$ is formed by points without preimages. The corresponding recurrence is called of type $Z_{0}-Z_{2}$ according to the Mira's classification [1].

The map $T$ has two inverse functions corresponding to the two antecedents points of the region $Z_{2}$, which are defined as follows:

$$
\begin{aligned}
& T_{1}^{-1}\left(x^{\prime}, y^{\prime}, z^{\prime}\right):\left\{\begin{array}{l}
x=\frac{-a}{2} x^{\prime}+\xi \\
y=x^{\prime} \\
z=y^{\prime}
\end{array}\right. \\
& T_{2}^{-1}\left(x^{\prime}, y^{\prime}, z^{\prime}\right):\left\{\begin{array}{l}
x=\frac{-a}{2} x^{\prime}-\xi \\
y=x^{\prime} \\
z=y^{\prime}
\end{array}\right.
\end{aligned}
$$

where $\xi=\frac{1}{2} \sqrt{a^{2} x^{\prime 2}-4 a x^{\prime} y^{\prime}+4 z^{\prime}-4 b}$.

These two functions are defined if $\frac{1}{4} a^{2} x^{\prime 2}-a x^{\prime} y^{\prime}+z^{\prime}-b \geqslant 0$. The condition $\frac{1}{4} a^{2} x^{\prime 2}-a x^{\prime} y^{\prime}+$ $+z^{\prime}-b \geqslant 0$ is verified for $x^{\prime}=y, y^{\prime}=z$ and $z^{\prime} \geqslant \frac{1}{4} a^{2} y^{2}+a y\left(-\frac{1}{2} a y+z\right)+b$. 
Hence the characterization of both regions $Z_{0}$ and $Z_{2}$ is given by:

$$
\begin{aligned}
& Z_{0}=\left\{(x, y, z) \in \mathbb{R}, a^{2} x^{2}-4 a x y+4 z-4 b<0\right\}, \\
& Z_{2}=\left\{(x, y, z) \in \mathbb{R}, a^{2} x^{2}-4 a x y+4 z-4 b>0\right\} .
\end{aligned}
$$

Each of the two inverse determinations is associated with two layers defined in the zone $Z_{2}$, which join along $E C$. We also define two unbounded regions $R_{1}$ and $R_{2}$ such that $E C_{-1}=R_{1} \cap R_{2}$ and for all $X$ belonging to $Z_{2}, T_{1}^{-1}(X) \in R_{1}$ and $T_{2}^{-1}(X) \in R_{2}$. When $X \in E C$, we get $T_{1}^{-1}(X)=T_{2}^{-1}(X)$ belonging to $E C_{-1}$ (Fig. 4).

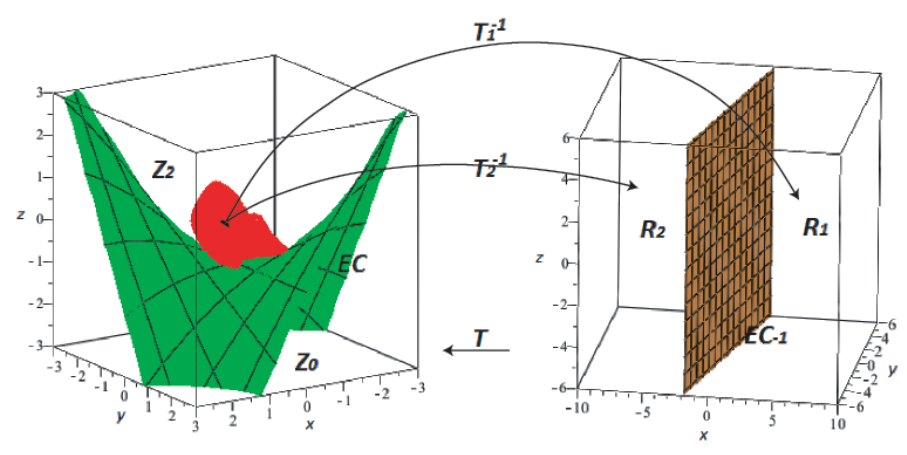

Fig. 4. Browsing $T$ and the regions $R_{1}$ and $R_{2}$ after application of inverse determinations of $T$. $E C$ and $E C_{-1}$ are obtained at $a=-0.6$ and $b=-0.75$

\subsection{Contact bifurcation}

Denote by $Q$ the stable fixed point $X_{2}$, and $\Gamma$ the invariant closed curve, consequent from the destabilization of the fixed point $Q \in R_{2}$, of type focus, via a Neimark-Sacker bifurcation. The curve is such that $\Gamma \subset R_{2}$ before the bifurcation exposed here, that is $\Gamma \cap E C_{-1}=\emptyset$ (see Fig. 5(a)).

The following properties hold (cf. [1] for particular example, [11] for the general case of $Z_{0}-Z_{2}$ maps and [3] for two-dimensional noninvertible maps):

Property 1. We have the following properties:

1. $\Gamma \cap E C_{-1}=\emptyset, \Gamma$ and its preimage of rank one $T_{1}^{-1}(\Gamma)=\Gamma_{-1}$ are separate and do not intersect, that is $\Gamma \cap \Gamma_{-1}=\emptyset$, with:

$$
T^{-1}(\Gamma)=T_{1}^{-1}(\Gamma) \cup T_{2}^{-1}(\Gamma)=\Gamma_{-1} \cup \Gamma
$$

2. The inner area bounded by $\Gamma$, denoted $a(\Gamma)$ with $Q \in a(\Gamma)$, is invariant by direct iteration $T(a(\Gamma))=a(\Gamma)$; and reverse determination of $\mathrm{T}$ defined in $a(\Gamma)$ is only given by $T_{2}^{-1}$. This characterizes the invariance of $a(\Gamma)$ and $\Gamma$ through the inverse iteration. Therefore, there is a subset $U$ of $\Gamma$ wherein $\mathrm{T}$ admit a unique inverse defined by $T_{2}^{-1}: U \longrightarrow U$. This is the local invariance iteration.

For two values of the parameters $a=\widehat{a}_{1}$ and $b=\widehat{b}_{1}$, a contact bifurcation appears when the invariant curve $\Gamma$ comes into contact with $E C_{-1}$ at a point noted $u_{0}\left(\Gamma \cap E C_{-1}=\left\{u_{0}\right\}\right)$; moreover, the image $u_{1}$ of $u_{0}$ by $\mathrm{T}$ is a point of tangential contact between $\Gamma$ and $E C$. For small values $\epsilon_{1}, \epsilon_{2}$ such that $a=\widehat{a}_{1}+\epsilon_{1}$ and $b=\widehat{b}_{1}+\epsilon_{2}$, we have:

$$
\Gamma \cap E C_{-1}=\left\{u_{0}, v_{0}\right\} \text { and } \Gamma \cap R_{1} \neq \emptyset,
$$




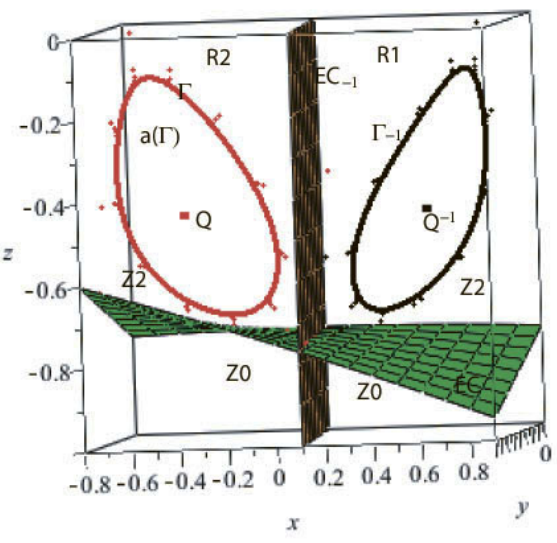

(a) $a=0.25$

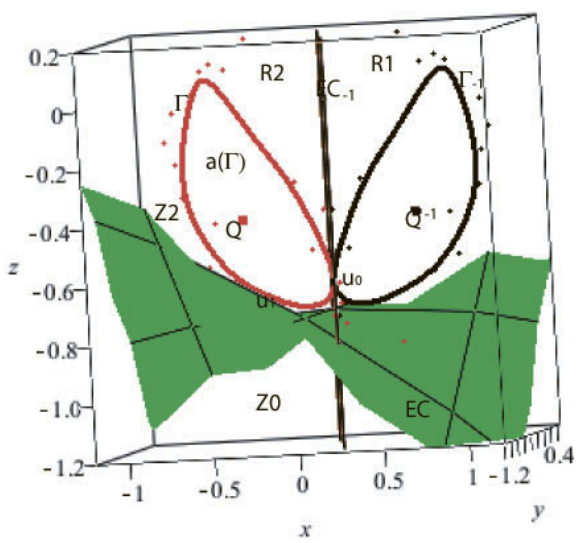

(b) $a=0.446$

Fig. 5. Before the bifurcation, there is no contact between the closed invariant curve $\Gamma$ and preimage of rank one $\Gamma_{-1}$; and after the bifurcation $\Gamma$ intersects $\Gamma_{-1}$ through $E C_{-1}$ and comes into tangential contact with $E C$ at $b=-0.75$ with $\left.X_{0}=(0.1,0.1,0.1)\right)$.

which means that $\Gamma$ intersects $E C_{-1}$ in two points $u_{0}$ and $v_{0}$; and invariant curve touches the zone $R_{1}$. This intersection causes the changes of the curve $\Gamma$ because the iterates of the rank $n$ for the couple $\left\{u_{0}, v_{0}\right\}$ noted $\left\{u_{n}, v_{n}\right\}_{n \geqslant 1}$ are points of contact between $\Gamma$ and $E C_{n-1}$. Indeed, $\Gamma$ cannot pass through all critical manifolds $\bigcup_{i=1}^{m-1} E C_{i-1}$ (all critical manifolds delimiting $\Gamma$, except $E C_{-1}$ ), which means in the opposite case that a point of $Z_{0}$ has an antecedent. This bifurcation creates oscillations of $\Gamma$ along the $E C_{m}$. Before the bifurcation, $\Gamma$ is invariant by the inverse iteration $T_{2}^{-1}$. After the bifurcation, the $\operatorname{arc}\left(u_{1}, v_{1}\right)$ of $\Gamma$ stays in $\Gamma$, neither by the inverse iteration $T_{2}^{-1}$ but by $T_{1}^{-1}$. The curve $\Gamma$ changes the sheet on $u_{1}$ and $v_{1}$ at the contact with $E C$ creating oscillations in the projected three-dimensional space (see Fig. 6).

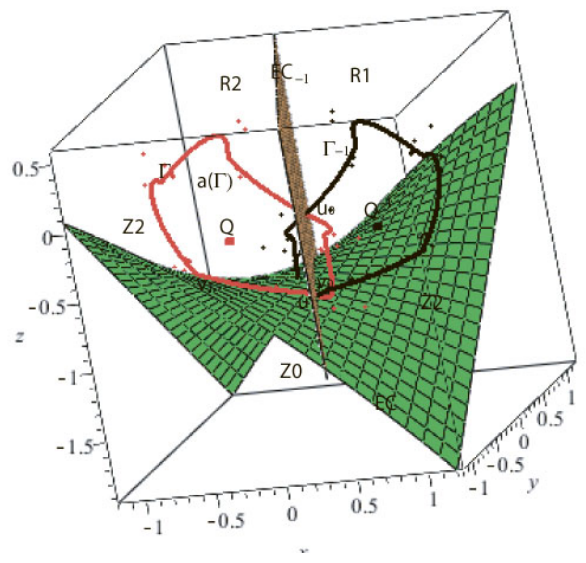

Fig. 6. After the bifurcation $\Gamma$ intersects $\Gamma_{-1}$ in two points $\left(u_{0}, v_{0}\right)$ through $E C_{-1}$ and comes into tangential contact with $E C$ in two points $\left(u_{1}, v_{1}\right)$ at $a=0.7$ and $b=-0.75$ with $X_{0}=$ $=(0.1,0.1,0.1)$

Property 2. Suppose that $\Gamma \cap E C_{-1} \neq \emptyset$ and $\Gamma \cap R_{1} \neq \emptyset$, then we have the following properties: 
1. The area $a(\Gamma)$ is no longer invariant by $T: T(a(\Gamma)) \neq a(\Gamma)$.

2. $\Gamma$ and $\Gamma_{-1}$ intersect at least on two points $u_{0}$ and $v_{0}$ of $E C_{-1}$. The part $\Gamma \subset R_{1}$ is folded and becomes tangent to the critical manifold $E C$ by direct iteration $T$.

3. $\Gamma$ is not invariant by inverse iteration. Indeed, the restricted inverse $T_{\Gamma}$ for the map $T$ in $\Gamma$ is defined now by $T_{1}^{-1}$ and $T_{2}^{-1}$ with respect to the considered part of $\Gamma$ and $T_{2}^{-1}(\Gamma) \neq \Gamma$.

4. There exists a neighborhood $U(\Gamma)$ of $\Gamma$, for which $T$ do not have a unique inverse from $U$ to $U$; it is defined by the two inverse determinations $T_{1}^{-1}$ and $T_{2}^{-1}$. This characterizes the local non invariance of $T$ by the inverse iteration.

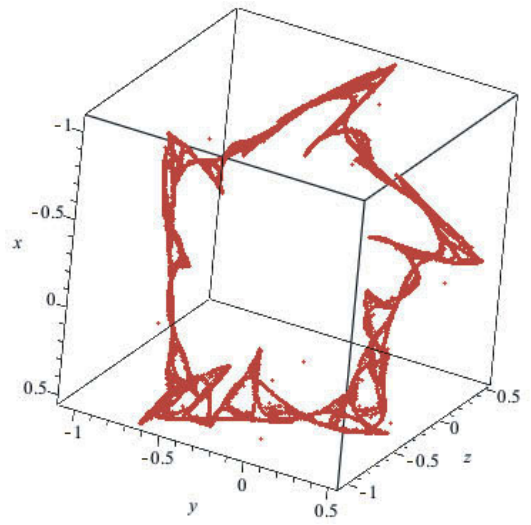

(a)

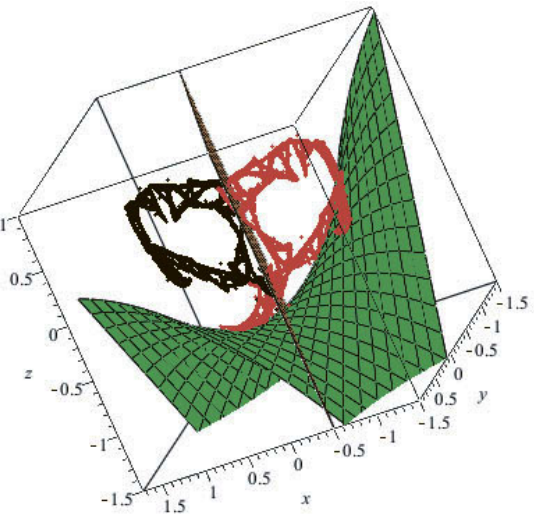

(b)

Fig. 7. Enlargement of the closed invariant curve at $a=1.01$ and $b=-0.75$ with $X_{0}=$ $=(0.1,0.1,0.1)$

For $a=\widehat{a}_{2}$ and $b=\widehat{b}_{2}$, the closed curve $\Gamma$ becomes tangent to $E C$ (see Fig. 7 ) and the some property of tangency occur in all the images of $E C$ (see Fig. 8), with the new change of sheets for $\Gamma$ in a point that a neighborhood $U$ verifies the fourth characterization of the Property 2.

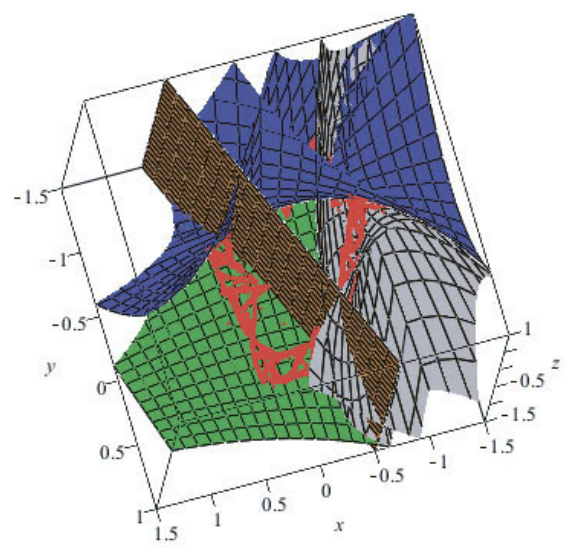

Fig. 8. Tangency of the closed invariant curve $\Gamma$ (in red color) with the critical surfaces $E C_{0}$ (green), $E C_{1}$ (blue) and $E C_{2}$ (gray) at $a=1.01$ and $b=-0.75$ with $X_{0}=(0.1,0.1,0.1)$ 
The bifurcation creating self-intersections on the invariant curve $\Gamma$ (points of $\Gamma \cap \Gamma_{-1} \in E C_{-1}$ ) causes the transformation of a closed invariant curve into a chaotic attractor. Indeed, for an closed invariant curve, the rotation's number associated is irrational, therefore there exist infinity points of self-intersections reproduced by self-similarity and successive iterations of $T$, which means that $\Gamma$ becomes fractal and its dimension $D_{L}$ in the sense of Lyapounov becomes higher (see Fig. 9(a)). Thus, the formed set is called weakly chaotic and by increasing parameters the complexity of the invariant curve $\Gamma$ grows. This results in an enlargement of the weakly chaotic attractor and increased Lyapounov exponents, as well as the associated $D_{L}$ dimension and this generates a chaotic attractor (see Fig. 9).

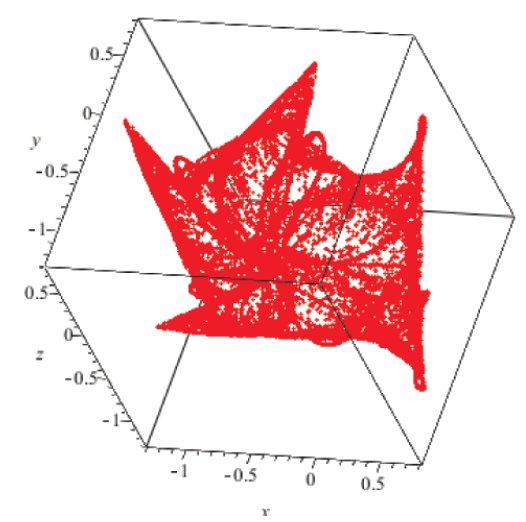

(a) $\mathrm{a}=1.12$

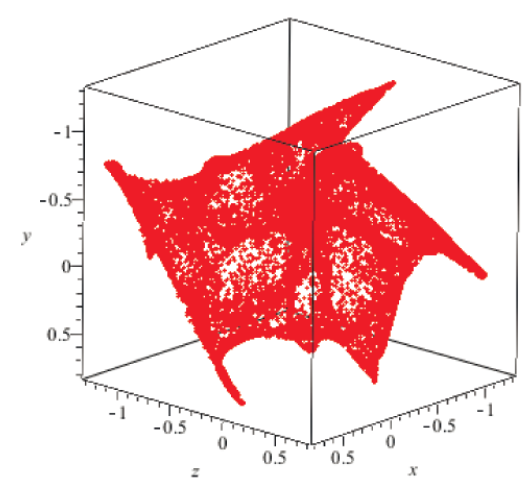

(c) $\mathrm{a}=1.14$

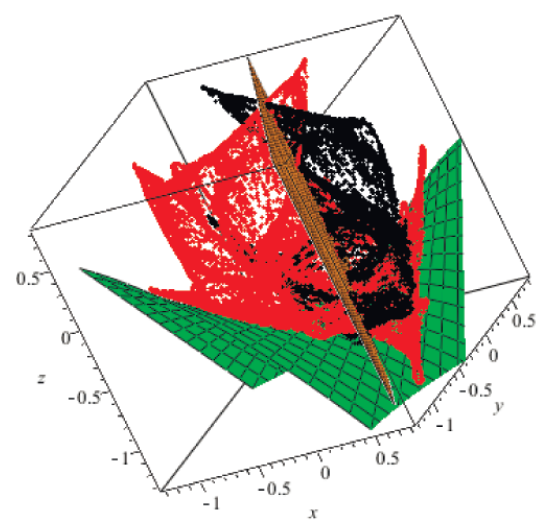

(b) $\mathrm{a}=1.12$

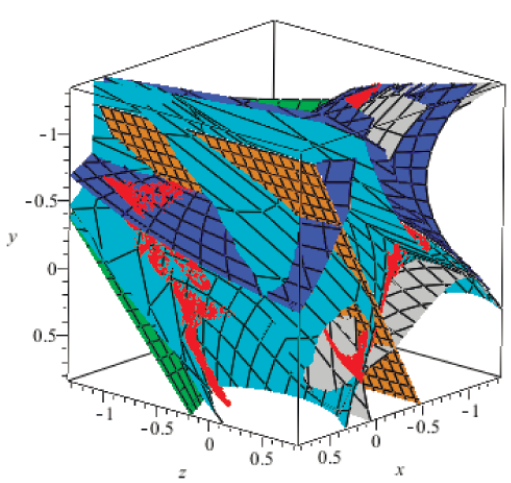

(d) $\mathrm{a}=1.14$

Fig. 9. Creation of the chaotic attractor at $b=-0.75$ with $X_{0}=(0.1,0.1,0.1)$. (a) Chaotic attractor in the space at $a=1.12$ and $b=-0.75$ and the corresponding Lyapounov dimension $D_{L}=2.12932$. (b) The attractor intersection with its antecedent of rank one, through $E C_{-1}$ and into tangential contact with $E C$. (c) Chaotic attractor in the space at $a=1.14$ and $b=-0.75$ and $\left.D_{L}=2.19071\right)$. (d) Chaotic attractor limited by the critical surfaces

\section{Study of $T$ with $b$ fixed and $a$ variable}

As we have already mentioned above, the transformation $T$ defined in (1) is of type $Z_{0}-Z_{2}$, has two fixed points $X_{1}$ and $X_{2}$, saddle focus and a stable node focus respectively for the values of $a$ and $b$ defined by the Neimark-Sacker, Flip and Fold bifurcation curves. If we vary the parameter 
$a$ in the positive direction with $b<-0.75$, the stable fixed point of map $T$ becomes unstable giving rise to an invariant closed curve via a Neimark-Sacker bifurcation for $(a, b)=(0,-0.75)$. However, if $a$ varies in the negative direction, the birth of two invariant closed orbits is observed. The existence of two dynamics is clearly illustrated by the bifurcation diagram (Fig. 10(a)) for different values of a and $b=-0.75$, showing the appearance of double orbits (negative sense of the variations of $a$ ) and orbits of order one (positive direction of the variations of $a$ ) are illustrated by the projection on the plane of the phases $(x, y)$, as well as the magnification given in Fig. 10(b). For $(a, b)=(0,-0.75)$ the transformation $\mathrm{T}$ has a fixed point of order one, which is the only fixed point of order two of $T$ and which belongs to the Neimark-Sacker bifurcation curve $\left(\mathrm{X}=(-0.5,-0.5,-0.5)\right.$ of multipliers $S_{1}=\overline{S_{2}}=0.5 \div 0.8660254038 I, S_{3}=-1$ and of modulus $\rho=1)$.

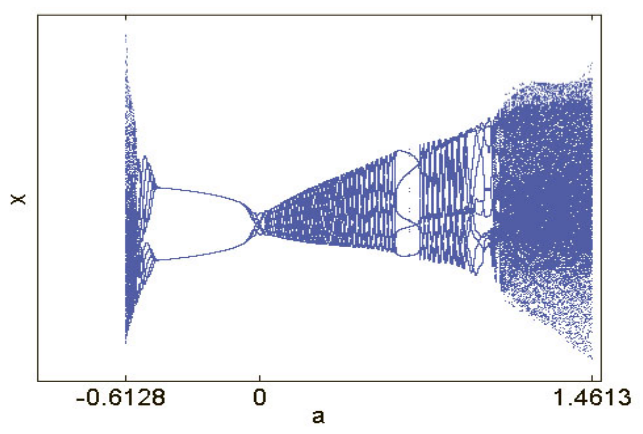

(a) Bifurcation diagram $(b=-0.85)$

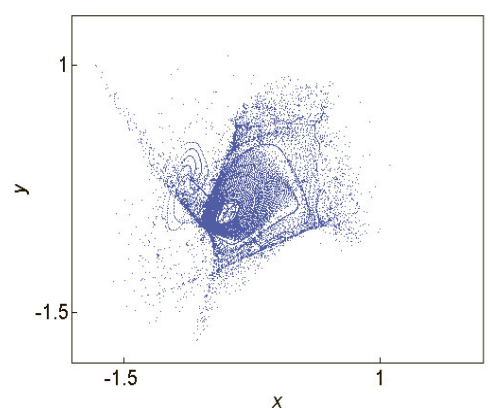

(b) Projection in the plane $(x, y)$

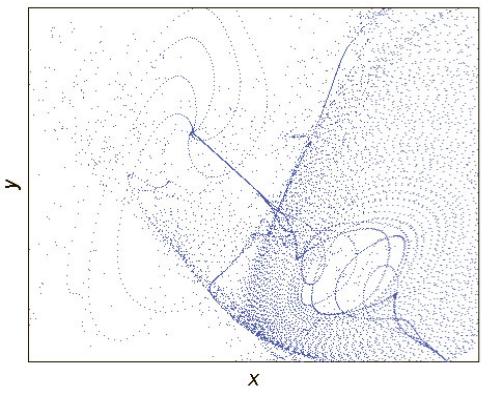

(c) Enlargement of the projection

Fig. 10. Bifurcation diagram at $b=-0.75$ and his projection in the phase plane $(x, y)$

Indeed, for $b=-0.75$ and by increasing $a$, the closed invariant curve $\Gamma$ comes into contact with the critical manifolds for the value $a=0.446$ (see Fig. 5(a)). Thus, oscillations of the invariant curve appear along the critical manifolds (see Figs. 6 and 7); for $a=1.01$ and $b=-0.75$ the self-intersections generate loops on $\Gamma$, which becomes a weakly chaotic attractor.

By increasing the parameter $a$, loops are formed and the set of points such that $\Gamma \cap \Gamma_{-1} \notin$ $E C_{-1}$ grows. The complexity of $\Gamma$ increases, and becomes a chaotic attractor (see Fig. 9(a) and 9(b)). We give in Fig. 9(c) the chaotic attractor obtained for $a=1.14$ and $b=-0.75$ and some critical manifolds which delimited and enveloped it (see Fig. 9(d)). The corresponding Lyapounov dimensions $D_{L}(a)$ are $D_{L}(1.01)=1.44203, D_{L}(1.12)=2.12932$ and $D_{L}(1.14)=2.19071$. By increasing the parameter $a$, the attractor comes into contact with the border of its attraction basin which disappears for $a=1.461391$ (see Fig. 11).

The two fixed points of the map $T^{2}$ are stable for $b=-0.75$ and $-0.484 \leqslant a<0$ becomes 
unstable for $a<-0.484$ and through a Neimark-Sacker bifurcation, to two closed invariant curves $\Gamma_{1}$ and $\Gamma_{2}$ appears in the zone $Z_{2}$, such that:

$$
\Gamma=\Gamma_{1} \cup \Gamma_{2} \text { and } T_{1}^{-1}(\Gamma) \cup T_{2}^{-1}(\Gamma)=\Gamma_{-1}^{1} \cup \Gamma_{-1}^{2} \text {. }
$$

For $a=-0.5$ and $b=-0.75$, the closed invariant curve $\Gamma_{1}$ comes into contact with $E C_{-1}$ and intersects its antecedent of rank one $\Gamma_{-1}^{1}$ on two points $u_{0}$ and $v_{0}$, once the bifurcation contact occurred; and the invariant curve $\Gamma_{2}$ comes into a tangential contact with $E C$ on two points $u_{1}$ and $v_{1}$, which are the images of $u_{0}$ and $v_{0}$ by direct application of map $\mathrm{T}$ (see Fig. 12(a)):

$$
\Gamma \cap E C_{-1}=\left\{u_{0}, v_{0}\right\}
$$

Varying the parameter $a$, even in the negative direction the invariant closed curves $\Gamma_{1}$ and $\Gamma_{2}$ change the form (each curve is folded and doubled). From then on, the closed invariant curve $\Gamma_{1}$ comes into contact with $\Gamma_{-1}^{1}$ on four points $u_{0}, v_{0}, r_{0}$ and $s_{0}$ belonging to $E C_{-1}$; and $\Gamma_{2}$ contacts $E C$ in four points $u_{1}, v_{1}, r_{1}$ and $s_{1}$ which are the images of the points $u_{0}, v_{0}, r_{0}$ and $s_{0}$ by $\mathrm{T}$ (see Fig. 12(b)).

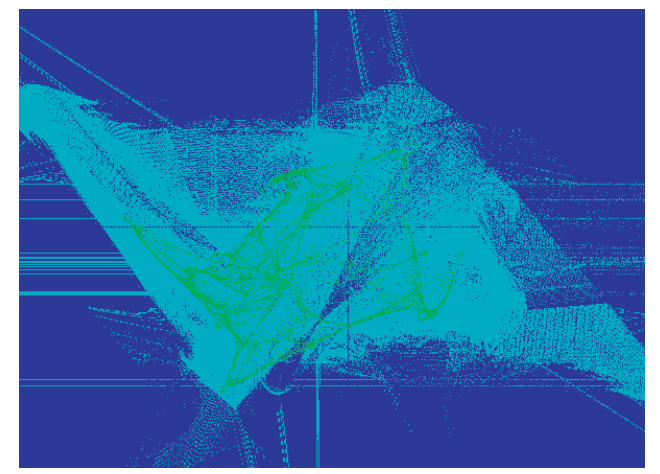

Fig. 11. The chaotic attractor (obtained at $a=1.14$ and $b=-0.75$ in color green) is going to undergo a contact bifurcation with its attraction basin boundary (in color cyan), which will make it disappear and which occurs at $a=1.461391$

For $a=-0.556$ and $b=-0.75$, the self-intersections generate loops on $\Gamma\left(\Gamma=\Gamma_{1} \cup \Gamma_{2}\right)$, which becomes a weakly chaotic attractor, with the Lyapounov dimension $D_{L}=2.00322$ (see Fig. 13(a)). Furthermore, if we reduce the parameter $a$, loops are formed and the set of points such that $\Gamma \cap \Gamma_{-1} \notin E C_{-1}$ grows. Then, the two cyclic attractors expand and merge into a single attractor. The complexity of $\Gamma$ and the Lyapounov dimension increase $\left(D_{L}(a): D_{L}(-0.56)=\right.$ 2.04589, $D_{L}(-0.6)=2.65599$ and $\left.D_{L}(-0.61)=3\right)$ (see Figs. 13(b) and 13(c)). Hence, the attractor becomes a chaotic. By increasing the parameter $a$, the attractor touches the border of his attraction basin and disappears for $a=-0.612$ (see Fig. 14).

\section{Study of $T$ with $a$ fixed and $b$ variable}

The coexistence of two attractors is observed when the parameter $a$ is fixed and $b$ is varied. The coexistence of two invariant curves for the map $T$ is illustrated by the Fig. 15 with $a=-0.1$ and $b$ varied $(b=-1.25$ and $b=-1.33)$. We showing that the two coexisting invariant curves in Fig. 15(a)), becomes a weakly chaotic attractors (Fig. 15(b)) after the bifurcation, before the merge in a single hyper-chaotic attractor at $a=-0.1$ and $b=-1.38$ (see Fig. 16(d)). 


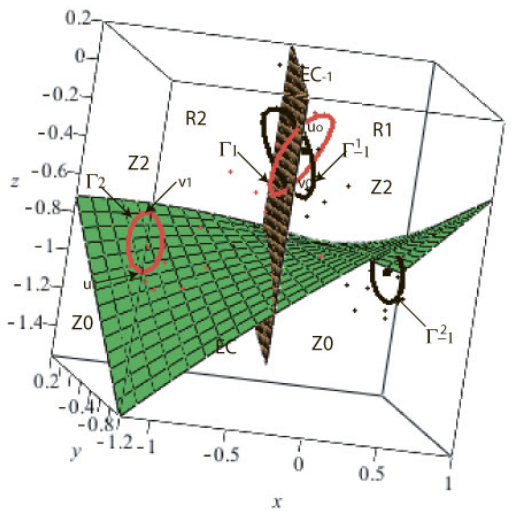

(a) $a=-0.5$

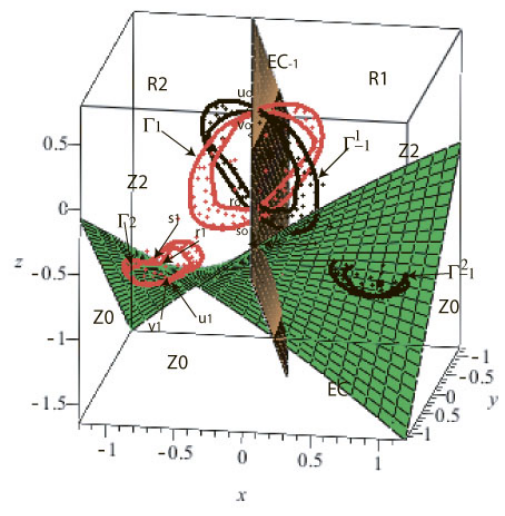

(b) $a=-0.546$

Fig. 12. Bifurcation contacts of two invariant closed curves with $E C_{-1}\left(b=-0.75, X_{0}=\right.$ $(0.1,0.1,0.1))$. (a) $\Gamma_{1}$ intersects $\Gamma_{-1}^{1}$ on two points $u_{0}$ and $v_{0}$ through $E C_{-1}$ and $\Gamma_{2}$ comes into a tangential contact with $E C$ on two points $u_{1}$ and $v_{1}$ (at $a=-0.5$ and $b=-0.75$ ). (b) Folding and doubling of the two invariant closed curves, $\Gamma_{1}$ intersects $\Gamma_{-1}^{1}$ on four points $u_{0}, v_{0}, r_{0}$ and $s_{0}$ belonging to $E C_{-1}$ and $\Gamma_{2}$ contacts $E C$ in four points $u_{1}, v_{1}, r_{1}$ and $s_{1}$ (at $a=-0.546$ and $b=-0.75)$

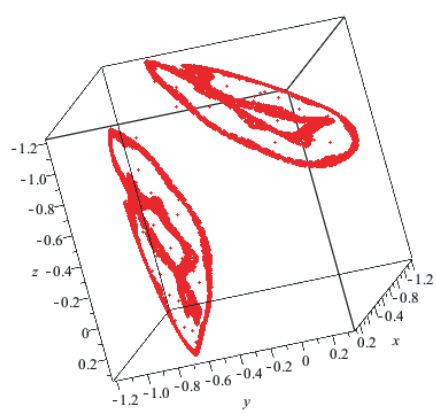

(a) $a=-0.556$

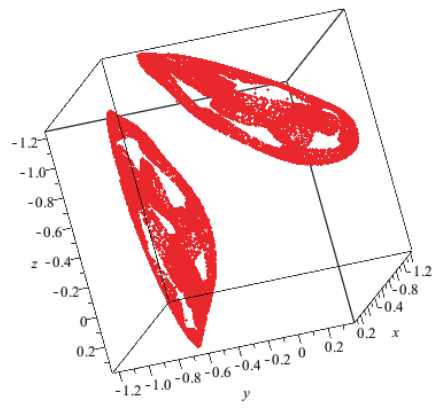

(b) $a=-0.56$

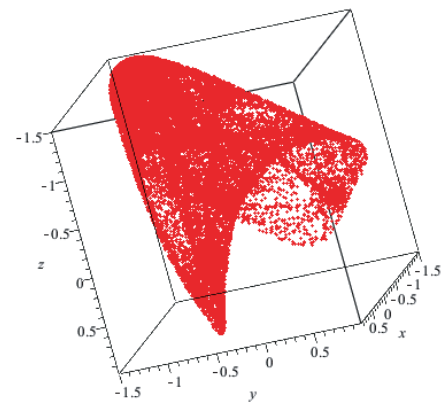

(c) $a=-0.6$

Fig. 13. Evolution of the two closed invariant curves into a chaotic attractor at $b=-0.75$ with $X_{0}=(0.1,0.1,0.1)$

- Suppose that $X_{0}=(0.1,0.1,0.1)$; the stable fixed point of the map $T$ becomes unstable and gives rise to an invariant closed curve via a Neimark-Sacker bifurcation $(b \approx-0.7732418029)$, which turns into a chaotic attractor via the bifurcations of contacts with the critical manifolds and the antecedent of rank one of this attractor. By decreasing the parameter $b$, oscillations are created on $\Gamma$ and Lyapounov dimension $D_{L}$ grows $\left(\right.$ e.g. $\left.D_{L}(-1.08)=2.00245\right)$, and an weakly chaotic attractor appears for $b=-1.1$ (see Fig. 17).

If we still decreasing the parameter $b$, the chaotic attractor disappears for $b \approx-1.1058$, and an attractor of six parts appears (see Fig. 16(a)). Furthermore, the attractor which is born for $b=-1.1058$ becomes weakly chaotic and the points number provided from the intersection of this attractor with his antecedent of rank one outside of $E C_{-1}$ increases, and its complexity also, as well as the Lyapounov dimension $\left(D_{L}(b): D_{L}(-1.2)=1.15288, D_{L}(-1.26)=1.47679\right.$ and $\left.D_{L}(-1.3)=2.08497\right)$. The attractor becomes chaotic and Lyapounov dimension DL reaches the 


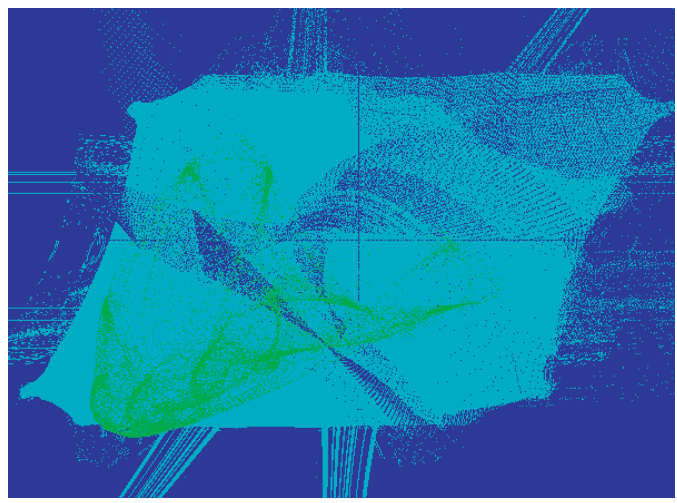

Fig. 14. The chaotic attractor (in color green) is going to undergo a contact bifurcation with its basin boundary (in cyan), which will make it disappear (figure for $a=-0.6$ and $b=-0.75$ before the contact bifurcation) and which occurs at $a=-0.612$

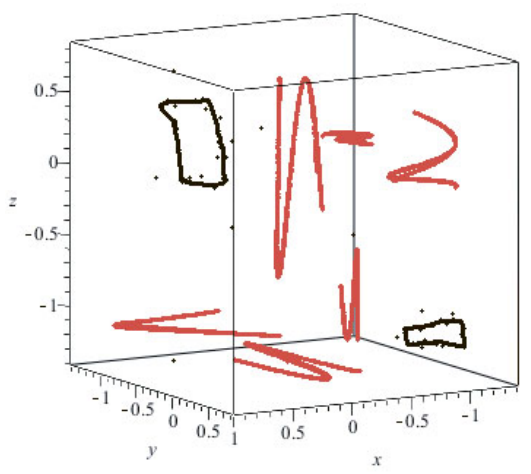

(a)

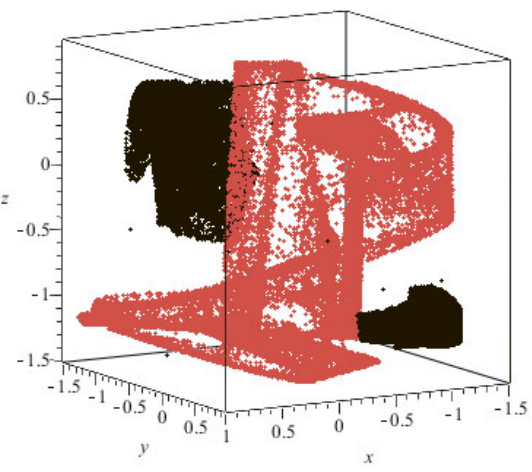

(b)

Fig. 15. Coexistence of two attractors. (a) Two invariant curves coexist for the map $T$ at $a=-0.1$ and $b=-1.25$. (b) The two invariant curves become chaotic attractors at $a=-0.1$ and $b=-1.33$

value of 3 and disappears for $b<-1.57879$ (see Fig. 16).

- By choosing $X_{0}=(1.5,1,-0.5)$, the stable cycle with the order two of the map $\mathrm{T}$ for $-1.155 \leqslant b<-0.7$, becomes unstable and gives rise to an invariant curve formed by two closed orbits (see Fig. 18(a)), which evolves to the chaotic attractor previously obtained (see Fig. 16(d)), through the contact bifurcations with the critical manifolds. Here we give some figures which show the qualitative changes of the invariant curve. By decreasing the parameter $b$, the invariant curve comes into contact with its antecedent of rank one with crossing the $E C_{-1}$ (see Fig. 18(b)), and oscillations are created.

By still decreasing $b$, a weakly chaotic attractor is formed for $b=-1.27$, and expands for $b=-1.34$ and $D_{L}(-1.34)=3$ (see Fig. 18(c)); then the two parts of the attractor merge and form the single chaotic attractor obtained at $b=-1.38$ (see Fig. 16(d)), whose Lyapounov dimension is $D_{L}(-1.38)=3$. The hyper chaotic attractor touches the border of his attraction basin and disappears at $b=-1.5788$. The attraction basins are illustrated for the attractors obtained at $b=-1.34$ and $b=-1.38$ with $a=-0.1$ in Fig. 19.

The figures of attraction basins and the Lyapounov dimension are obtained using the Dynamics logiciel [12]. 


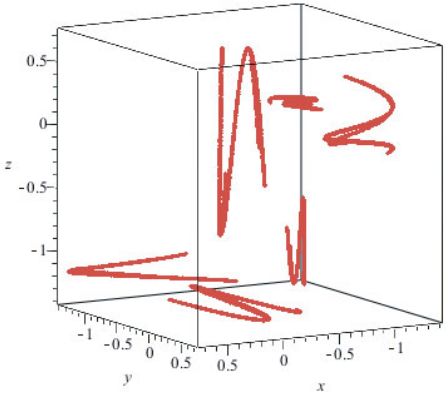

(a) $b=-1.0$

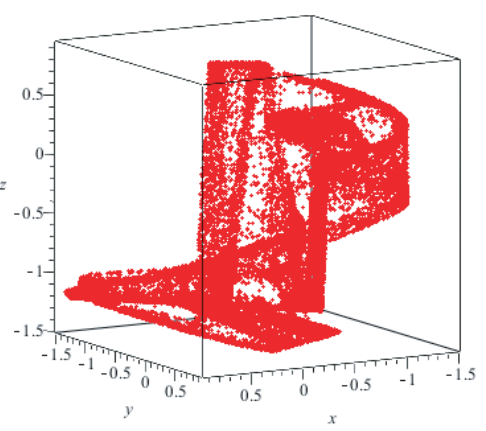

(c) $b=-1.1$

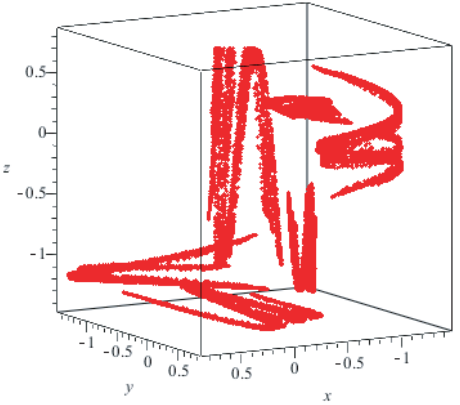

(b) $b=-1.1$

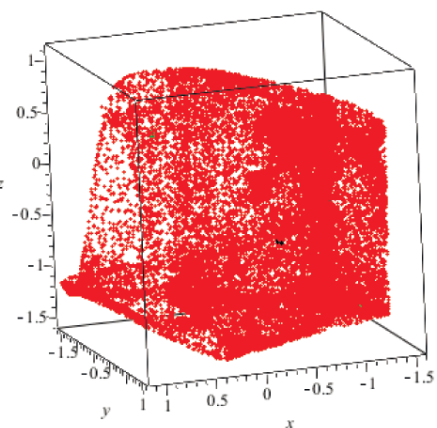

(d) $b=-1.1$

Fig. 16. The second chaotic dynamics for $a=-0.1$ and $X_{0}=(0.1,0.1,0.1)$

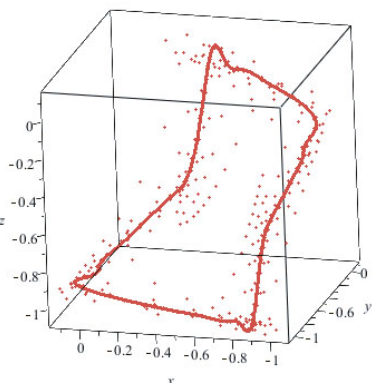

(a) $b=-1$.

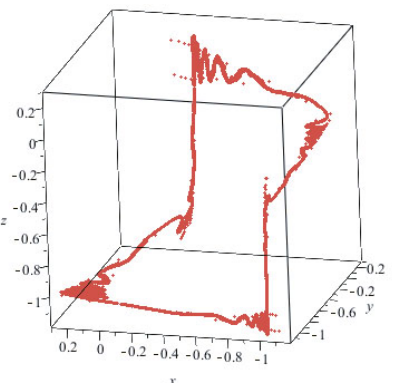

(b) $b=-1.08$

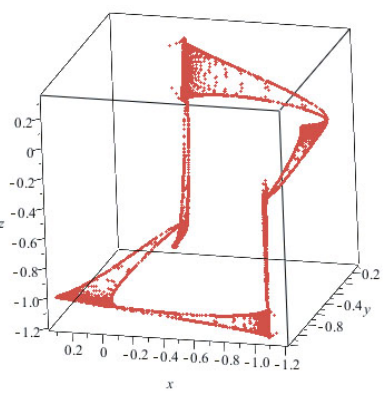

(c) $b=-1.1$

Fig. 17. The dynamic evolution of a closed invariant curve to chaotic attractor $(a=-0.1$, $\left.X_{0}=(0.1,0.1,0.1)\right)$

\section{Conclusion}

In this work, by setting one of the parameters $(a$ and $b)$ and varying the other parameter of the endomorphism $T$, the dynamic evolutions of some attractors have been illustrated in the phase space. The passage from invariant curves or attractors to weakly chaotic attractors then to hyper-chaotic attractors via contact bifurcations with critical varieties (critical surfaces) in the three-dimensional phase space, which disappear after the bifurcation of contact with the limit of their basins of attraction. The coexistence of invariant orbits or attractors is observed as well as their dynamic evolutions towards a single chaotic attractor. 


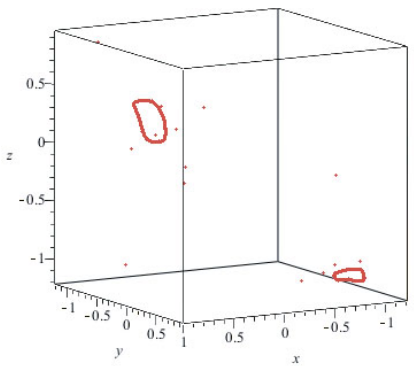

(a) $b=-1.19$.

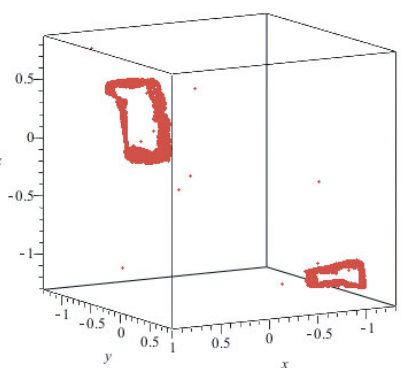

(b) $b=-1.27$

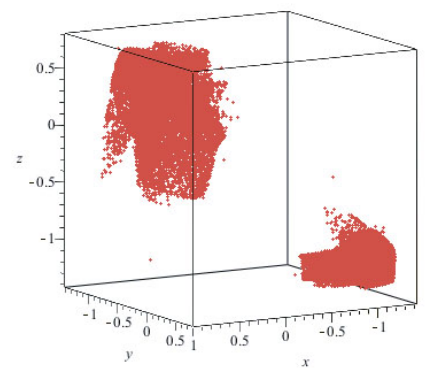

(c) $b=-1.34$.

Fig. 18. Evolution of two invariant closed curves to a weakly chaotic attractor, then the two parts of the attractor merge and form a single hyper chaotic attractor (obtained at $b=-1.38$ and illustrated in Fig. 16(d))); at $a=-0.1$ with $X_{0}=(1.5,1,-0.5)$

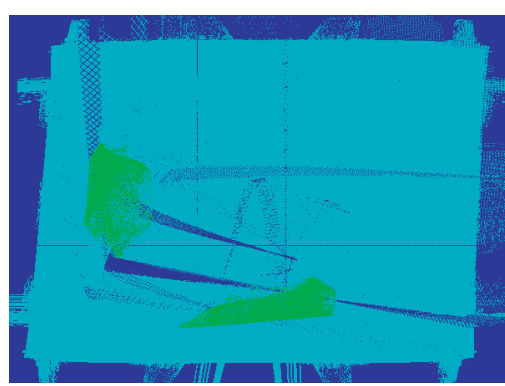

(a)

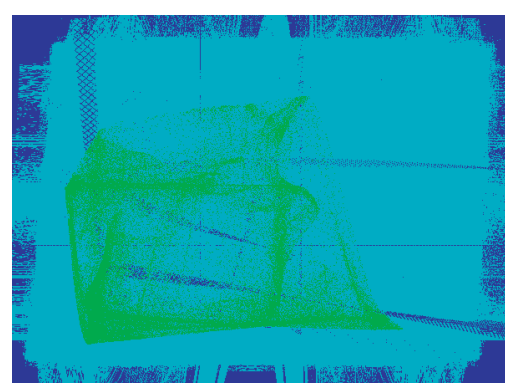

(b)

Fig. 19. (a) The attraction basin (in color cyan) of the weakly chaotic attractor obtained at $a=-0.1$ and $b=-1.34$ with $X_{0}=(1.5,1,-0.5)$. (b) The chaotic attractor (at $a=-0.1$ and $b=-1.38$ in color green) and its attraction basin (in cyan), which disappears after the contact bifurcation with the its basin boundary which occurs at $b=-1.5788$

\section{References}

[1] I.Gumowski, C.Mira, Dynamique chaotique Transformatios ponctuelles Transition OrdreDésordre, Cepadues Editions, Toulouse, 1980.

[2] C.Mira, Chaotic Dynamics: From the One-dimensional Endomorphism to the TwoDimensional Diffeomorphism, World Scientific Publishing, Singapore, 1987.

[3] C.E.Frouzakis, L.Gardini, I.G.Kevrekidis, G.Millerioux, C.Mira, On some proporties of invariant sets of two-dimensional noninvertible maps, International Journal of Bifurcation and Chaos, 7(1997), no. 7, 1167-1194.

[4] C.Mira, A.Agliari, L.Gardini, From the box-within-a-box bifurcation structure to the julia set. Part II: Bifurcation routes to different julia sets from an indirect embedding of a quadratic complex map, International Journal of Bifurcation and Chaos, 19(2009), no. 10, $3235-3282$.

[5] G.Millerioux, C.Mira, Noninvertible piecewise linear maps applied to chaos synchronization and secure communications, International Journal of Bifurcation and Chaos, 7(1997), no. 7, $1617-1634$. 
[6] E.Mosekilde, Y.Maistrenko, D.Postnov, Chaotic Synchronization: Applications to living systems, World Scientific Series on Nonlinear science, Series A, vol. 42, Singapore, 2001.

[7] J.Xu, D.Fournier-Prunaret, A.K.Taha, P.Chargé, Chaos generator for secure transmission using a sine map and a RLC series circuit, Science China, 1(2010), no. 53, 129-136.

[8] J.C.Sprott, Simplest dissipative chaotic flow, Physics Letters A, 228(1997), 271-274.

[9] G.M.Zaslavsky, Chaos in Dynamic Systems, Harwood, Amsterdam, 1985.

[10] G.I.Bischi, L.Mroz, H.Hauser, Studying basin bifurcations in nonlinear triopoly games by using 3D visualization, Nonlinear Analysis, Theory, Methods \& Applications, 47(2001), no. $8,5325-5341$.

[11] C.Mira, L.Gardini, A.Barugola, J.C.Cathala, Chaotic dynamics in two-dimensional noninvertible maps, World Scientific Publishing. Serie A, Singapore, 1996.

[12] H.E.Nusse, J.A.Yorke, Dynamics: Numerical Explorations, Springer-Verlag, New York, 1998.

\title{
Хаотическая динамика трехмерного эндоморфизма
}

\author{
Хасен Гаро \\ Ноуредин Акроун \\ Факультет точных наук \\ Университет Бежа, Бежа, 06000 \\ Алжир \\ Абелкадоус Таха \\ Рангуил, 135, Тулуза, 31077 \\ Франция
}

Национальный институт прикладных наук Тулузы

Даниел-Фоурниер Прунаре

Национальный институт прикладных наук Тулузы

Полковник Рош-авеню, 7, Тулуза, 31077

Франция

В настоящей работе описываются бифуркации фазовых плоскостей некоторых аттракторов, задаваемых необратимым трехмерным отображением. Это исследование проводится с помощъю концепций критических многообразий, обобщения критических точек и критических линий, введенных Гумовским и Мирой [1, 2]. Фазовая плоскость делится в двух открытых областях: первая (обозначается Z0) каждая точка, не имеющая реального прообраза, а вторая (обозначенная Z2) каждая точка имеет два реальных прообраза. Области Z0, Z2 разделены критическими многообразиями, локус точек, имеющих два совпадающих прообраза. Для этого требуется визуализация критических многообразий в пространстве трехмерных фаз. Работа также описывает прохождение инвариантных или аттракторных кривых в сторону слабохаотических аттракторов, а затем $к$ гиперхаотическим аттракторам через контактную бифуркацию, через критические многообразия, которые исчезают после контактной бифуркачии с ее границей притяжения.

Ключевые слова: критическое многообразие, замкнутая инвариантная кривая, слабый хаос, хаос. 